\title{
Misclassification of child body mass index from cut-points defined by rounded percentiles instead of Z-scores
}

\author{
Laura N. Anderson 1,2* (1), Sarah Carsley ${ }^{2,6}$, Gerald Lebovic ${ }^{3,6}$, Cornelia M. Borkhoff ${ }^{2,6,7}$, Jonathon L. Maguire $3,4,5,6$, \\ Patricia C. Parkin 2,5,6,7 and Catherine S. Birken ${ }^{2,5,6,7}$
}

\begin{abstract}
Objective: To evaluate the misclassification resulting from the use of body mass index (BMI) cut-points defined by rounded percentiles instead of Z-scores in early childhood. Using data from the TARGet Kids primary care network we conducted a cross-sectional study among 5836 children $<6$ years of age. The World Health Organization growth standards were used to calculate BMI-for-age Z-scores. BMI Z-score cut-points of $<-2.0,>1.0,>2.0,>3.0$ are recommended to define wasted, at risk of overweight, overweight and obese. However, rounded percentiles of the $3 \mathrm{rd}$, 85th, 97th, and 99.9th are commonly used. Misclassification was calculated comparing the frequency distributions for BMI categories defined by rounded percentiles and Z-score cut-points.
\end{abstract}

Results: Using rounded percentiles, the proportion of children who were wasted, at risk of overweight, overweight, and obese was 4.2, 12.5, 4.3 and 0.8\%, whereas the distribution using Z-scores was: 3.6, 13.8, 3.4 and 1.0\%, respectively. Overall, 117 (2\%) children were misclassified when using percentiles instead of Z-scores; however, 13\% (33/245) of children who were wasted and 14\% (8/57) of children who were obese were misclassified. Misclassification of child growth results from the use of cut-points defined by rounded percentiles instead of Z-scores and limits comparability between studies.

Trial registration Clinicaltrials.gov NCT01869530 June 5, 2013

Keywords: Growth charts, Body mass index, Pediatric obesity, Child, preschool, Validation studies

\section{Introduction}

Monitoring of child growth, including classification of both underweight and overweight categories, is important for population health, and routine child growth monitoring is recommended [1]. The World Health Organization (WHO) Child Growth Standards charts are recommended as a reference standard for the growth monitoring of children $0-5$ years of age and have been endorsed by many countries $[2,3]$. The WHO Growth Standards were developed following children from 6 countries under optimal growth conditions [4].

\footnotetext{
*Correspondence: LN.Anderson@mcmaster.ca

${ }^{1}$ Department of Health Research Methods, Evidence, and Impact,

McMaster University, 1280 Main Street West, Hamilton, ON L8N 3Z5,

Canada

Full list of author information is available at the end of the article
}

Regardless of which reference standard is used, cut-point definitions are required for clinicians and researchers to classify children's growth status. These cut-points for age and sex standardized growth measures are defined using either Z-scores or percentiles.

The WHO Child Growth Standards provide child growth measures standardized by age and sex using Z-scores. Z-scores, or standard deviation scores, describe where an observation falls within a number of standard deviations of the mean. For example, body mass index (BMI)-for-age Z-score cut-points of $<-2.0,>1.0,>2.0$ and $>3.0$ are recommended by the WHO to classify children $0-5$ years of age as wasted, risk-of-overweight, overweight, and obese, respectively [5]. Several advantages of the Z-score for population-based assessment of child growth have been described [6]. Z-scores are on a 
linear scale, with the same interval between values across the distribution; this allows for calculation of the mean and standard deviation [7].

In contrast, percentiles rank a child's position in comparison to a reference population. Z-scores can be converted directly to percentiles [8], although when converted the percentiles are often rounded. Rounded percentiles of the 3rd, 85th, 97th and 99.9th are commonly used in clinical practice and recommended for monitoring child growth in Canada [2]. These rounded percentiles correspond to exact Z-scores of $-1.88,1.04,1.88$ and 3.09, in comparison to the WHO recommended cutpoints of $-2.0,1.0,2.0$ and 3.0, respectively. Z-scores have been recommended for research, and percentiles for clinical settings as they may be easier to understand [7].

However, the use of cut-points defined by rounded percentiles instead of exact $\mathrm{z}$-scores may result in misclassification of children's growth status, yet to the best of our knowledge, the magnitude of this misclassification has not been evaluated. This may be important when comparing differences between studies that have unintentionally used slightly different cut-points. The objective of this study was to evaluate the degree of misclassification resulting from the use of cut-points defined by rounded percentiles relative to Z-scores for growth monitoring in early childhood.

\section{Main text}

\section{Study design}

A cross-sectional study was conducted among children $0-5$ years. Children were recruited from scheduled well-child primary care visits through TARGet Kids!. TARGet Kids! is a primary care practice based research network (http://www.targetkids.ca) [9, 10]. Children were recruited from nine primary care pediatricians or family practice clinics in Toronto, Canada between 2008 and 2016. Children with severe developmental delay or chronic illness (except for asthma) were excluded.

\section{Anthropometric measures}

Trained research team members measured child weight and height (or length for children $<2$ years). Weight was measured using a precision digital scale (SECA, Germany). A stadiometer was used to measure standing height (SECA) for children 2 years of age and older and length was measured for children under 2 years of age using a length board [9]. Weight in kilograms was divided by the height (or length) in meters squared to calculate BMI.

Age and sex standardized BMI Z-scores were defined using the World Health Organization (WHO) growth standards [11]. The WHO growth standards were selected as they are meant to reflect optimal growth in children and are recommended for use in this age group in Canada [2]. BMI-for-age Z-score cut-points of $<-2.0,>1.0,>2.0$ and $>3.0$ were used to classify children as wasted, risk of overweight, overweight and obese, respectively [2]. BMI-for-age Z-scores were categorized using both the Z-score cut-points of $<-2.0,>1.0,>2.0$ and $>3.0$, and rounded percentile cut-points of 3rd, 85th, 97th and 99.9th, which correspond to exact Z-scores of $-1.88,1.04,1.88$ and 3.09 .

\section{Statistical analysis}

Cross tabulations of the number of children for each category were calculated comparing the two definitions (Z-score cut-points and rounded percentile cut-points) and discordant and concordant categories were reported. The percent misclassified was calculated both overall from the total and within growth categories. The relative percent misclassified within each category was calculated by dividing the difference between the two definitions (\% defined by percentile minus Z-score cut-point) by the total percent defined by the Z-score cut-point. Kappa and weighted Kappa statistics were calculated as a measure of agreement. Although BMI is standardized for age and sex using the WHO growth standards, we further evaluated whether there were differences in misclassification by sex using stratified analysis.

\section{Results}

There were 5836 unique children who participated in this study. The mean age of children was 28 months $(\mathrm{SD}=19), 2770(48 \%)$ were female and $3066(53 \%)$ were male. The distribution of maternal ethnicity was 3818 (67\%) European, 361 (7\%) East Asian, 544 (10\%) South or South East Asian, 277 (5\%) African and 580 (11\%) other or mixed ethnicity. The median neighborhood household income was $\$ 55,038(\mathrm{SD}=\$ 25,484)$.

A total of 117 (2\%) children were misclassified when using percentiles instead of Z-scores (Table 1). Within the extreme growth categories $13 \%$ (33/245) of children who were wasted and $14 \%(8 / 57)$ of children who were obese were misclassified by the use of rounded percentiles instead of Z-scores. Further, the relative percent misclassified for wasted, risk of overweight, overweight and obese was $17,-9,26$ and $-20 \%$, respectively, when using percentiles instead of Z-score cut-points (Fig. 1). Agreement between the two methods was high with a Kappa coefficient of 0.95 (95\% CI 0.94, 0.96) and weighted Kappa of 0.96 (95\% CI 0.95, 0.97).

The results were similar when boys and girls were examined separately; a total of $68(2.2 \%)$ boys and 49 (1.8\%) girls were misclassified. The overall misclassification was slightly greater in boys than girls and this may be explained by the fact that more boys than girls were 
Table 1 Agreement between growth categories using Z-score and percentile cut-points for classification of BMI categories in children $0-5$ years of age $(n=5836)$

\begin{tabular}{|c|c|c|c|c|c|}
\hline \multirow[t]{3}{*}{ Z-score cut-points } & \multicolumn{5}{|c|}{ Percentile cut-points } \\
\hline & Wasted & Normal & Risk of overweight & Overweight & Obese \\
\hline & $<3 r d$ & 3rd to 85th & $>85$ th to 97 th & $>97$ th to 99.9 th & $>$ 99.9th \\
\hline$<-2.0$ & $212(3.6 \%)$ & 0 & 0 & 0 & 0 \\
\hline-2.0 to 1.0 & $33(0.6 \%)$ & $4530(77.6 \%)$ & 0 & 0 & 0 \\
\hline$>1.0$ to 2.0 & 0 & $37(0.6 \%)$ & $727(12.5 \%)$ & $39(0.7 \%)$ & 0 \\
\hline$>2.0$ to 3.0 & 0 & 0 & 0 & $201(3.4 \%)$ & 0 \\
\hline$>3.0$ & 0 & 0 & 0 & $8(0.1 \%)$ & $49(0.8 \%)$ \\
\hline
\end{tabular}

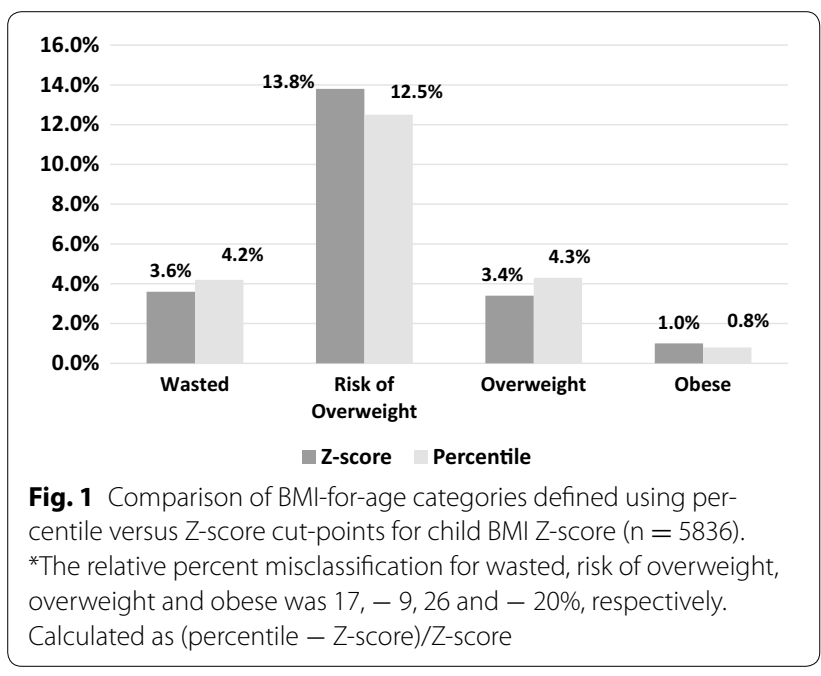

classified in the extreme growth categories. For example, using the Z-score cut-points, the percent of boys classified as wasted, risk of overweight, overweight and obese was $4.0,14.4,4.0$ and $1.3 \%$ respectively, whereas the percent of girls was 3.1, 13.0, 2.8, $0.7 \%$.

\section{Discussion}

In this study of 5836 children $0-5$ years of age we found misclassification from the use of zBMI cut-points defined by rounded percentiles instead of Z-scores. The overall degree of misclassification was small at only $2 \%$ of all children. However, within the extreme growth categories there was substantial misclassification, for example among children classified as wasted an additional 17\% were misclassified, and among children classified as obese $20 \%$ were missed. Strengths of our study include the large sample size and standardized measurement of heights/ lengths and weights by trained research assistants.

Recent population-based reports have used inconsistent cut-point definitions which we have shown may limit comparability for the extreme growth categories. For example, some studies have used percentile cut-points
[12, 13], whereas in other studies Z-score cut-points have been used $[14,15]$. It is possible that researchers are using Z-score cut-points in analysis and labeling them as percentiles for perceived ease of interpretation but this is not clear. We suggest that the terms not be used interchangeably or assumed that results would be the same.

Percentiles may be useful when interpreting values for individual children for both parents and health professionals, however, the WHO recommends that researchers use Z-scores for consistency and comparability. The use of percentiles also causes problems due to limited range especially at the extreme ends for example one proposed definition of severe obesity in young children is 1.2 times the 95th percentile. Due to the misclassification that results when cut-points defined using rounded percentiles and Z-scores are compared and the limitations of percentiles, we suggest that future data analysis is performed using Z-score cut-points and not percentiles [8]. To the best of our knowledge, we are the first to evaluate the misclassification from rounded percentiles versus Z-score cut-points.

\section{Limitations}

One limitation of this study is that we were not able to evaluate the validity of each approach in relation to a gold-standard. We were unable to evaluate the clinical implications of this misclassification. Further, we were unable to estimate how frequently this misclassification occurs in the literature as it is often not clear which approach was used in previous studies. The choice of cut-points may not matter when comparing repeated measures over time within one population or within one child, but the observed differences may be very important when comparing the prevalence of growth categories across different studies or populations (e.g., for public health surveillance). Others may argue for the use of continuous variables only due to inherent limitations of the use of any cut-points such as reduced power and loss of information. However, cut-points are often useful when 
interpreting results, defining high-risk individuals and are frequently used for both research and for clinical purposes. The validity and reliability of BMI-for-age in early childhood have been evaluated elsewhere [16-18].

Although the overall degree of misclassification from the use of cut-points defined by rounded percentiles compared to Z-scores was small, substantial misclassification was observed within the extreme growth categories. This misclassification may have important implications when comparing both prevalence estimates for population surveillance and risk estimates from research studies. This misclassification may substantially impact on policy and planning. Future guidelines should clearly recommend the use of either Z-scores or rounded percentiles for consistency in child growth monitoring.

\section{Abbreviations}

BMI: body mass index; WHO: World Health Organization.

\section{Authors' contributions}

LNA, CSB, CMB and SC conceived and designed the study. LNA, SC, CMB and $G L$ analyzed and interpreted the data. JLM, PCP and CSB obtained funding for the study and supervised data collection. LNA wrote the paper. All authors read and approved the final manuscript.

\section{Author details \\ ${ }^{1}$ Department of Health Research Methods, Evidence, and Impact, McMaster University, 1280 Main Street West, Hamilton, ON L8N 3Z5, Canada. ${ }^{2}$ Child Health Evaluative Sciences, The Hospital for Sick Children Research Institute, Peter Gilgan Centre for Research \& Learning, 686 Bay Street, Toronto, ON M5G OA4, Canada. ${ }^{3}$ The Applied Health Research Center of the Li Ka Shing Knowl- edge Institute of St. Michael's Hospital, 209 Victoria St, Toronto, ON M5B 1T8, Canada. ${ }^{4}$ Department of Nutritional Sciences, Faculty of Medicine, University of Toronto, FitzGerald Building, 150 College Street, Room 316, Toronto, ON M5S 3E2, Canada. ${ }^{5}$ Department of Pediatrics, Faculty of Medicine, University of Toronto, 555 University Avenue, Toronto, ON M5G 1X8, Canada. ${ }^{6}$ Institute for Health Policy, Management, and Evaluation, University of Toronto, Health Sciences Building, 155 College Street, Suite 425, Toronto, ON M5T 3M6, Canada. ${ }^{7}$ Division of Pediatric Medicine, The Hospital for Sick Children, 555 University Avenue, Toronto, ON M5G 1X8, Canada.}

\section{Acknowledgements}

We thank all of the participating families for their time and involvement in TARGet Kids! and are grateful to all practitioners who are currently involved in the TARGet Kids! practice-based research network.

\section{Competing interests}

The authors declare that they have no competing interests.

\section{Availability of data and materials}

Data are available upon request by contacting http://www.targetkids.ca/ contact-us/. The full data are not freely available to respect the confidentiality of our participants, ensure data integrity, and avoid scientific overlap between projects. Once initial contact has been made, we request a short research proposal which will be subject to review by the TARGet Kids! Scientific Committee and ethics approval.

\section{Consent for publication}

Not applicable.

\section{Ethics approval and consent to participate}

Informed written consent was obtained from parents of all participating children and ethical approval was obtained from the Research Ethics Board at The Hospital for Sick Children and St. Michael's Hospital.

\section{Funding}

This work was supported by research grants from the Canadian Institutes of Health Research.

\section{Publisher's Note}

Springer Nature remains neutral with regard to jurisdictional claims in published maps and institutional affiliations.

Received: 21 September 2017 Accepted: 22 November 2017

Published online: 28 November 2017

\section{References}

1. Canadian Task Force on Preventive Health Care. Recommendations for growth monitoring, and prevention and management of overweight and obesity in children and youth in primary care. CMAJ. 2015;187(6):411-21.

2. Secker D. Promoting optimal monitoring of child growth in Canada: using the new WHO growth charts. Can J Diet Pract Res. 2010;71(1):e1-3.

3. International Pediatric Association. Endorsement of the new WHO growth standards for infants and young children; 2006. http://www.who. int/childgrowth/Endorsement_IPA.pdf.

4. WHO Multicentre Growth Reference Study Group. WHO Child Growth Standards based on length/height, weight and age. Acta Paediatr. 2006;450:76-85.

5. de Onis M, Lobstein T. Defining obesity risk status in the general childhood population: which cut-offs should we use? Int J Pediatr Obes. 2010:5(6):458-60

6. World Health Organization. Global database on child growth and malnutrition. In: de Onis M, Blossner M, editors. Chapter 5.3: the Z-score or standard deviation classification system. Geneva: World Health Organization; 1997.

7. Wang Y, Chen HJ. Use of percentiles and z-scores in anthropometry. In: Preedy VR, editor. Handbook of anthropometry. New York: Springer; 2012. p. 29-48.

8. Must A, Anderson SE. Body mass index in children and adolescents: considerations for population-based applications. Int J Obes (Lond). 2006;30(4):590-4

9. Anderson LN, Lebovic G, Hamilton J, Hanley AJ, McCrindle BW, Maguire $J$, Parkin PC, Birken CS, Collaboration TK. Body mass index, waist circumference, and the clustering of cardiometabolic risk factors in early childhood. Paediatr Perinat Epidemiol. 2016;30(2):160-70.

10. Carsley S, Borkhoff CM, Maguire JL, Birken CS, Khovratovich M, McCrindle B, Macarthur C, Parkin PC, TARGet Kids collaboration. Cohort profile: the applied research group for kids (TARGet Kids!). Int J Epidemiol. 2015:44(3):776-88

11. de Onis M, Garza C, Victora CG. The WHO Multicentre Growth Reference Study: strategy for developing a new international growth reference. Forum Nutr. 2003;56:238-40.

12. Shields M, Tremblay MS. Canadian childhood obesity estimates based on WHO, IOTF and CDC cut-points. Int J Pediatr Obes. 2010;5(3):265-73.

13. Rodd C, Sharma AK. Recent trends in the prevalence of overweight and obesity among Canadian children. CMAJ. 2016;188(13):E313-20.

14. Black RE, Victora CG, Walker SP, Bhutta ZA, Christian P, de Onis M, Ezzati M, Grantham-McGregor S, Katz J, Martorell R, et al. Maternal and child undernutrition and overweight in low-income and middle-income countries. Lancet. 2013:382(9890):427-51.

15. Ahrens W, Pigeot I, Pohlabeln H, De Henauw S, Lissner L, Molnár D, Moreno LA, Tornaritis M, Veidebaum T, Siani A, et al. Prevalence of overweight and obesity in European children below the age of 10. Int J Obes (Lond). 2014;38(Suppl 2):S99-107.

16. Furlong KR, Anderson LN, Kang H, Lebovic G, Parkin PC, Maguire JL, O'Connor DL, Birken CS, Collaboration TAK. BMI-for-age and weight-forlength in children 0 to 2 years. Pediatrics. 2016;138(1):e20153809.

17. Roy SM, Spivack JG, Faith MS, Chesi A, Mitchell JA, Kelly A, Grant SF, McCormack SE, Zemel BS. Infant BMI or weight-for-length and obesity risk in early childhood. Pediatrics. 2016;137(5):e20153492.

18. WHO Multicentre Growth Reference Study Group. Reliability of anthropometric measurements in the WHO Multicentre Growth Reference Study. Acta Paediatr. 2006:450:38-46. 